\title{
Protective effect of probiotics on Salmonella infectivity assessed with combined in vitro gut fermentation-cellular models
}

\author{
Annina Zihler ${ }^{\dagger}$, Mélanie Gagnon ${ }^{\dagger}$, Christophe Chassard and Christophe Lacroix ${ }^{*}$
}

\begin{abstract}
Background: Accurate assessment of probiotics with targeted anti-Salmonella activity requires suitable models accounting for both, microbe-microbe and host-microbe interactions in gut environments. Here we report the combination of two original in vitro intestinal models closely mimicking the complex in vivo conditions of the large intestine. Effluents from continuous in vitro three-stage fermentation colonic models of Salmonella Typhimurium infection inoculated with immobilized child microbiota and Salmonella were directly applied to confluent mucussecreting HT29-MTX cell layers. The effects of Salmonella, addition of two bacteriocinogenic strains, Bifidobacterium thermophilum RBL67 (thermophilicin B67) and Escherichia coli L1000 (microcin B17), and inulin were tested on Salmonella growth and interactions with epithelial cell layers. Salmonella adhesion and invasion were investigated and epithelial integrity assessed by transepithelial electrical resistance (TER) measurements and confocal microscopy observation. Data from complex effluents were compared with pure Salmonella cultures.

Results: Salmonella in effluents of all reactors of the colonic fermentation model stabilized at mean values of $5.3 \pm$ $0.8 \log _{10} \mathrm{cfu} / \mathrm{ml}$ effluent. Invasion of cell-associated Salmonella was up to 50-fold lower in complex reactor samples compared to pure Salmonella cultures. It further depended on environmental factors, with $0.2 \pm 0.1 \%$ being measured with proximal, $0.6 \pm 0.2 \%$ with transverse and $1.3 \pm 0.7 \%$ with distal reactor effluents, accompanied by a similar high decrease of TER across cell monolayers (minus 45\%) and disruption of tight junctions. Subsequent addition of E. coli L1000 stimulated Salmonella growth (6.4 $\pm 0.6 \log _{10} \mathrm{cfu} / \mathrm{ml}$ effluent of all 3 reactors) and further decreased TER, but led to 10-fold decreased invasion efficiency when tested with distal reactor samples. In contrast, presence of $B$. thermophilum RBL67 revealed a protective effect on epithelial integrity compared to previous $E$. coli L1000 periods, as reflected by a significant mean increase of TER by 58\% in all reactors. Inulin addition enhanced Salmonella growth and invasion when tested with distal and proximal reactor samples, respectively, but induced a limited decrease of TER (minus 18\%) in all reactors.
\end{abstract}

Conclusions: Our results highlight the benefits of combining suitable cellular and colonic fermentation models to assess strain-specific first-level host protection properties of probiotics during Salmonella infection, providing an efficient system biology tool for preclinical development of new antimicrobials.

\section{Background}

The human colon constitutes a protective and nutrientrich habitat to trillions of bacteria living in symbiosis with the host [1]. This complex consortium constantly competes with exogenous microbes for attachment sites in the brush border of intestinal epithelial cells, thus

\footnotetext{
* Correspondence: christophe.lacroix@ilw.agrl.ethz.ch

† Contributed equally

Laboratory of Food Biotechnology, Institute of Food, Nutrition and Health,

ETH Zürich, Schmelzbergstrasse 7, 8092 Zürich, Switzerland
}

preventing pathogens from entering specific ecological niches and gut tissues [2]. Pathogens may however overcome this line of defense, leading to different manifestations of disease. Infectious gastroenteritis caused by non-typhoidal strains of Salmonella enterica spp. enterica is an important cause of morbidity and mortality worldwide [3]. Due to the increasing incidence of antibiotic resistant and more virulent serovars [4], the use of probiotics with specific anti-Salmonella activities is a prevailing interest. Mechanisms by which probiotics

C Biomed Central

(C) 2011 Zihler et al; licensee BioMed Central Ltd. This is an Open Access article distributed under the terms of the Creative Commons Attribution License (http://creativecommons.org/licenses/by/2.0), which permits unrestricted use, distribution, and reproduction in any medium, provided the original work is properly cited. 
inhibit pathogens include competition for nutritional substrates and adhesion sites on intestinal epithelial cells, secretion of antimicrobial substances as well as toxin inactivation and host immunity stimulation [5]. However, in vivo mechanistic studies of probiotics and gut microbiota are hindered by ethical considerations, compliance issues and high costs. A variety of in vitro gut models have been applied to separately investigate microbe-microbe and simple microbe-host interactions [6-8]. Owing to the complexity of the intestinal environment, suitable models accounting for all intestinal parameters including both the gut microbiota and their substrates and metabolic products as well as the presence of epithelial intestinal cells, represent an indispensable platform for preclinical probiosis assessment.

To investigate the complex gut microbiota in vitro, continuous intestinal fermentation models utilizing immobilized fecal microbiota have been developed for the controlled long-term cultivation of gut microbiota with conserved biodiversity [9-11]. Such models allow independent testing of different experimental treatments on both gut microbiota composition and metabolic activity within a single experimental period, using the same microbiota under controlled environmental conditions, which are designed to simulate the proximal, transverse and distal colon of healthy and infected subjects [9-14]. More recently, a three-stage in vitro colonic fermentation model of Salmonella infection in child colon was used to assess the effects of probiotic and prebiotic treatments on gut microbial behavior and on $S$. Typhimurium infection [15]. The activity of microcin B17-producing Escherichia coli L1000 wt [16] and bacteriocinogenic Bifidobacterium thermophilum RBL67, both exhibiting strong anti-Salmonella activity in simple in vitro tests $[17,18]$, as well as the microcin B17-negative mutant strain $M c c B 17-$, were tested in two threestage models inoculated with the same fecal inoculum. When added to the colonic model, E. coli L1000 unexpectedly stimulated Salmonella growth in all reactors independently of the microcin B17-phenotype, partly due to a low colonization of the strain in the complex intestinal environment. In contrast, thermophilicin RBL67-producing Bifidobacterium thermophilum RBL67 revealed high competitiveness and colonized at high levels but did not reduce Salmonella counts, most likely a function of the presence of a very high Salmonella population in the in vitro model prior to probiotic addition.

Most data available on the mechanistic effects of probiotics on the host are derived from in vitro studies with intestinal cells [19]. Such models have also been used to investigate bacterial interactions with the intestinal epithelium during enteric infection [20]. Salmonella pathogenesis, for example, has been studied in pure cultures using epithelial Caco-2 and HT-29 cell models $[21,22]$, both of which lack the ability to produce mucus. The mucus-secreting HT29-MTX cell line however, represents more accurate physiological conditions of the gastrointestinal tract for investigating pathogenic behavior during infection, as the presence of mucus has been shown to enhance pathogenicity of pathogens such as Campylobacter jejuni [23]. All interaction studies of pathogens and probiotics with intestinal cells have been performed with simple systems of either pure or mixed cultures. Microbe cell interactions are however different when tested in the presence of a complex gut microbiota [24,25]. Gut metabolites such as SCFAs affect epithelial cell metabolism, turnover and apoptosis [26] but may also enhance virulence (e.g. S. Typhimurium), by inducing an acid tolerance response or increasing expression of porins [27]. To our knowledge, the effects of an infected gut microbiota, including its metabolites and probiotic treatment on intestinal cells has not been previously reported.

In this study, the mucus-producing HT29-MTX cell model was used to investigate the interaction of $S$. Typhimurium N-15 in presence of a complex intestinal microbiota and to assess the host-protection properties of E. coli L1000 and B. thermophilum RBL67 sequentially inoculated in the infection model, as well as the protective effect of inulin. Effluent samples were produced in two three-stage continuous colonic models, mimicking the proximal, transverse and distal colon regions and inoculated with immobilized child fecal microbiota and Salmonella, and used to test the effects of probiotics and inulin on gut microbiota composition and metabolism, and on Salmonella growth [15]. Effluents collected from different fermentation periods were directly applied to HT29-MTX cells to measure Salmonella invasion and monitor changes in cellular integrity through both measurement of transepithelial electrical resistance (TER) and confocal microscopy. Data from complex effluents were compared with pure Salmonella cultures.

\section{Results}

Complex reactor effluents were collected during pseudosteady states (last 3 days) of different experimental periods from two continuous three-stage colonic fermentation models as indicated in Figure 1 and applied directly onto confluent mucus-secreting HT29-MTX cells. Temporal and environmental factors affecting bacterial growth, Salmonella invasion and TER across cell monolayers are summarized in Figure 2 and Table 1. TER across cell monolayers after incubation with simple and complex fermentation samples are compared in Figure 3 and the effects on epithelial integrity upon effluent application are shown in Figure 4. 


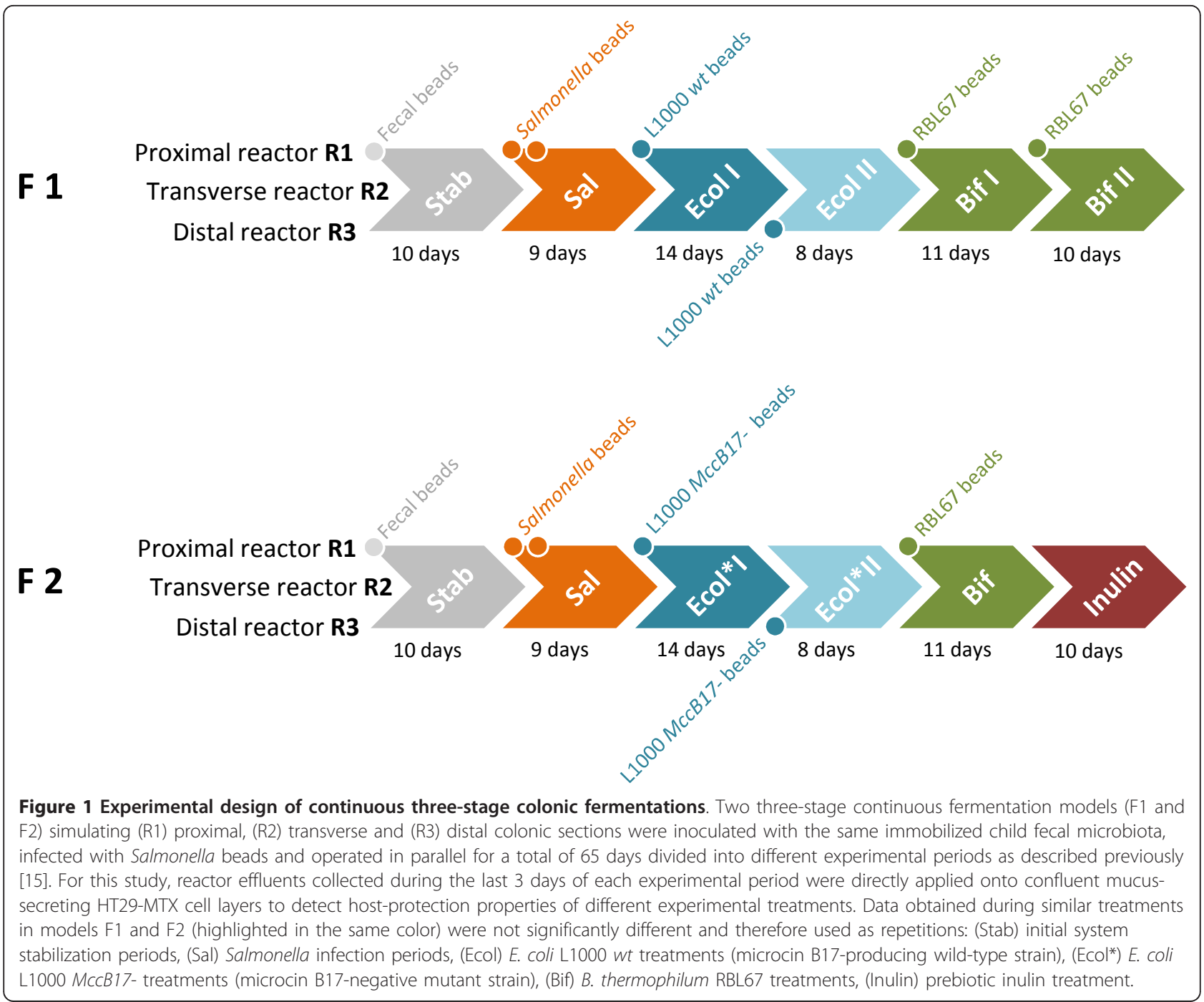

\section{Complex reactor effluents affect TER across HT29-MTX monolayers}

Salmonella were detected neither in reactor effluents nor after invasion assays in samples obtained at the end of initial model stabilization periods (Stab). Mean TER across HT29-MTX monolayers measured after 1-3 h incubation with effluents from initial model stabilization periods (Stab) were consistent and similar for all reactors $\left(251 \pm 23 \Omega \mathrm{cm}^{2}\right)$. Furthermore cellular tight junctions were unaffected after 90 min of incubation, as also demonstrated by confocal microscopy for distal reactor effluents of F1 (Figure 4A). 24 h post-incubation, a significant decrease of TER was recorded (Figure 3). A significantly $(P<0.05)$ higher TER was measured with transverse and distal effluents compared to proximal reactor effluents (Table 1), correlating with significantly increased SCFA concentrations in both R2 (177 \pm 6 $\mathrm{mM})$ and $\mathrm{R} 3(187 \pm 20 \mathrm{mM})$ compared to $\mathrm{R} 1(141 \pm 7$ $\mathrm{mM}$, Table 1).
Salmonella invasion is a function of environmental factors and affects epithelial integrity

Upon infection of the three-stage continuous fermentation model with $S$. Typhimurium N-15 beads (Sal, Figure 2A), Salmonella concentrations in effluents steadily increased and stabilized at significantly $(P<0.01)$ higher levels in proximal $\left(5.8 \pm 0.3 \log _{10} \mathrm{cfu} / \mathrm{ml}\right)$ and transverse $\left(5.6 \pm 0.5 \log _{10} \mathrm{cfu} / \mathrm{ml}\right)$ compared to distal colon reactors $\left(4.5 \pm 0.7 \log _{10} \mathrm{cfu} / \mathrm{ml}\right)$. Invasion efficiency expressed as percentage of cell-associated Salmonella, was significantly higher with effluents of R2 $(0.6 \pm 0.2 \%$; $P=0.049)$ and R3 $(1.3 \pm 0.7 \% ; P=0.002)$ compared to R1 $(0.2 \pm 0.1 \%)$ [Sal, Figure $2 \mathrm{C}$ ]. In contrast, invasion efficiency of pure cultures of Salmonella in buffered DMEM was up to 50 -fold higher $(9.8 \pm 2.1 \%)$.

Compared to non-infected effluents from initial model stabilization periods (Stab), a large and significant mean decrease of TER across HT29-MTX cell monolayers was measured after $1 \mathrm{~h}$ of incubation with effluents of all 


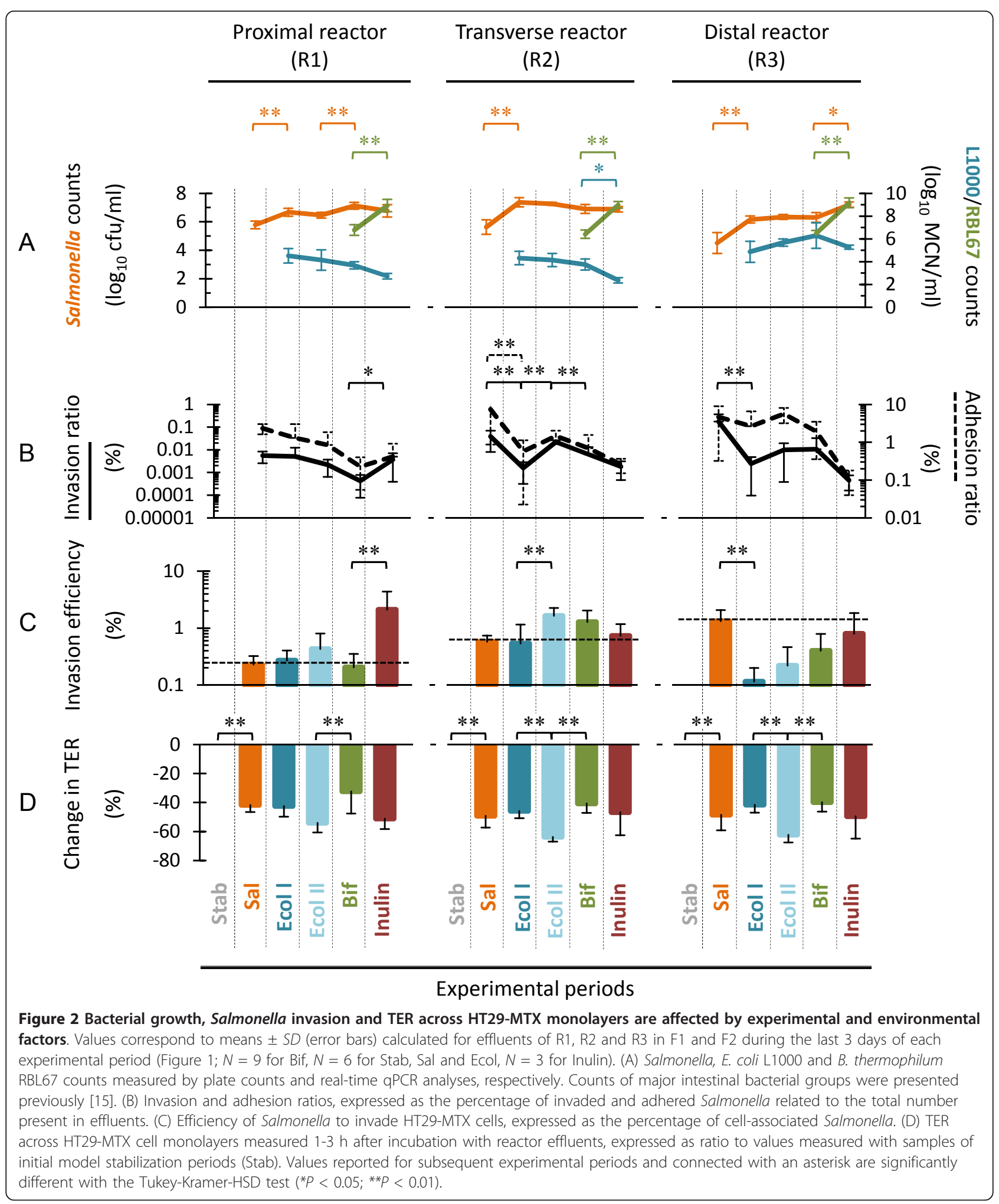

reactors from Salmonella infection periods (Sal, Figure 3). Mean TER values did not differ after 1-3 h of incubation $(P>0.05)$, but significantly decreased after $24 \mathrm{~h}$ of incubation (Figure 3). In contrast, TER measured for pure cultures of $S$. Typhimurium N-15 in buffered DMEM showed a continuous and pronounced decrease in TER (Figure 3). Compared to initial model stabilization periods (Stab), mean TER measured 1-3 h after 
Table 1 TER across HT29-MTX monolayers depends on temporal and environmental factors including SCFAs in reactor effluents

\begin{tabular}{|c|c|c|c|c|c|c|c|}
\hline & & \multicolumn{6}{|c|}{ Experimental period } \\
\hline & & Stab & Sal & Ecol I & Ecol II & Bif & Inulin \\
\hline \multicolumn{8}{|c|}{ R1 } \\
\hline \multirow[t]{2}{*}{$\overline{T E R}$} & $1-3 \mathrm{~h}$ & $247 \pm 24^{a}$ & $144 \pm 24^{\mathrm{bc}}$ & $143 \pm 22^{\mathrm{bc}}$ & $114 \pm 14^{c}$ & $167 \pm 34^{b}$ & $121 \pm 13^{b c}$ \\
\hline & $24 \mathrm{~h}$ & $127 \pm 23^{a}$ & $69 \pm 20^{b}$ & $55 \pm 11^{b}$ & $36 \pm 4^{b}$ & $130 \pm 47^{a}$ & $65 \pm 14^{b}$ \\
\hline \multirow[t]{2}{*}{$\overline{S C F A s} s^{*}(A: P: B)$} & & & & $\begin{array}{c}138 \pm 6^{a} \\
(54: 11: 34) \\
\end{array}$ & & & $\begin{array}{c}179 \pm 6^{a} \\
(44: 7: 50) \\
\end{array}$ \\
\hline & & & & & & & \\
\hline \multirow[t]{2}{*}{$\overline{T E R}$} & $1-3 \mathrm{~h}$ & $266 \pm 19^{\mathrm{a}}$ & $135 \pm 29^{b}$ & $144 \pm 17^{b}$ & $96 \pm 4^{c}$ & $158 \pm 8^{b}$ & $142 \pm 29^{b}$ \\
\hline & $24 \mathrm{~h}$ & $205 \pm 34^{\mathrm{a}}$ & $74 \pm 17^{c}$ & $52 \pm 4^{\text {cd }}$ & $34 \pm 8^{d}$ & $115 \pm 19^{b}$ & $87 \pm 11^{b c}$ \\
\hline \multirow[t]{2}{*}{$\overline{\mathrm{SCFAs}}(A: P: B)$} & & & & $\begin{array}{l}172 \pm 6^{b} \\
(54: 14: 32)\end{array}$ & & & $\begin{array}{l}245 \pm 6^{b} \\
(45: 12: 43)\end{array}$ \\
\hline & & & & & & & \\
\hline \multirow[t]{2}{*}{$\overline{T E R}$} & $1-3 \mathrm{~h}$ & $240 \pm 24^{a}$ & $124 \pm 30^{b c}$ & $141 \pm 16^{b}$ & $91 \pm 6^{c}$ & $145 \pm 8^{b}$ & $121 \pm 30^{\mathrm{bc}}$ \\
\hline & $24 \mathrm{~h}$ & $190 \pm 37^{a}$ & $75 \pm 17^{c d}$ & $77 \pm 13^{c}$ & $32 \pm 11^{d}$ & $119 \pm 30^{b}$ & $91 \pm 25^{b c}$ \\
\hline$\overline{S C F A s} s^{*}(A: P: B)$ & & & & $\begin{array}{c}180 \pm 13^{b} \\
(55: 14: 31)\end{array}$ & & & $\begin{array}{c}234 \pm 11^{b} \\
(46: 11: 43)\end{array}$ \\
\hline
\end{tabular}

Mean transepithelial electrical resistance (TER; expressed in $\left.\Omega \mathrm{cm}^{2}\right) \pm S D$ were measured after incubation of HT29-MTX cell monolayers for 1-3 $\mathrm{h}(\mathrm{N}=18)$ and 24 h $(N=6)$ with effluents retained from (R1) proximal, (R2) transverse and (R3) distal colon reactors of F1 and F2 during the last three days of each experimental period. Values with different letters in a row of the same reactor are significantly different according to the Tukey-Kramer-HSD test $(P<0.05)$. ${ }^{*}$ No treatment effects (except for inulin addition) were detected on total short chain fatty acid (SCFA) concentrations (expressed in mM). Mean SCFA concentrations \pm SD and (A) acetate: $(P)$ propionate: $(B)$ butyrate ratios measured during the last three days of non-inulin $(N=33)$ and inulin $(N=3)$ periods are therefore presented. Values with different letters in the same column of different reactors are significantly different with the Tukey-Kramer-HSD test $(P<0.05)$. (Stab) initial system stabilization periods, (Sal) Salmonella infection periods, (Ecol) E. coli L1000 treatments, (Bif) B. thermophilum RBL67 treatments, (Inulin) prebiotic inulin treatment.

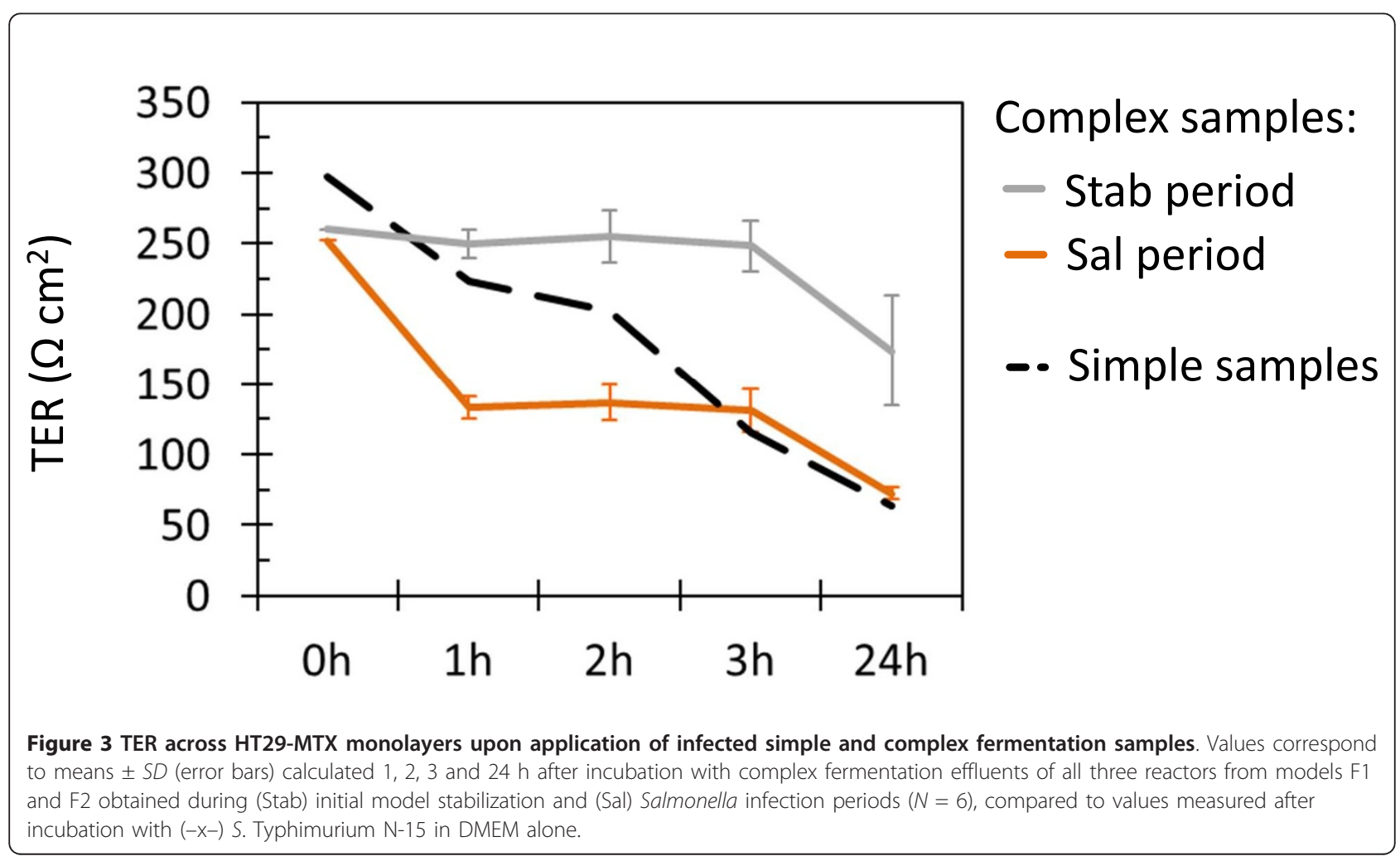



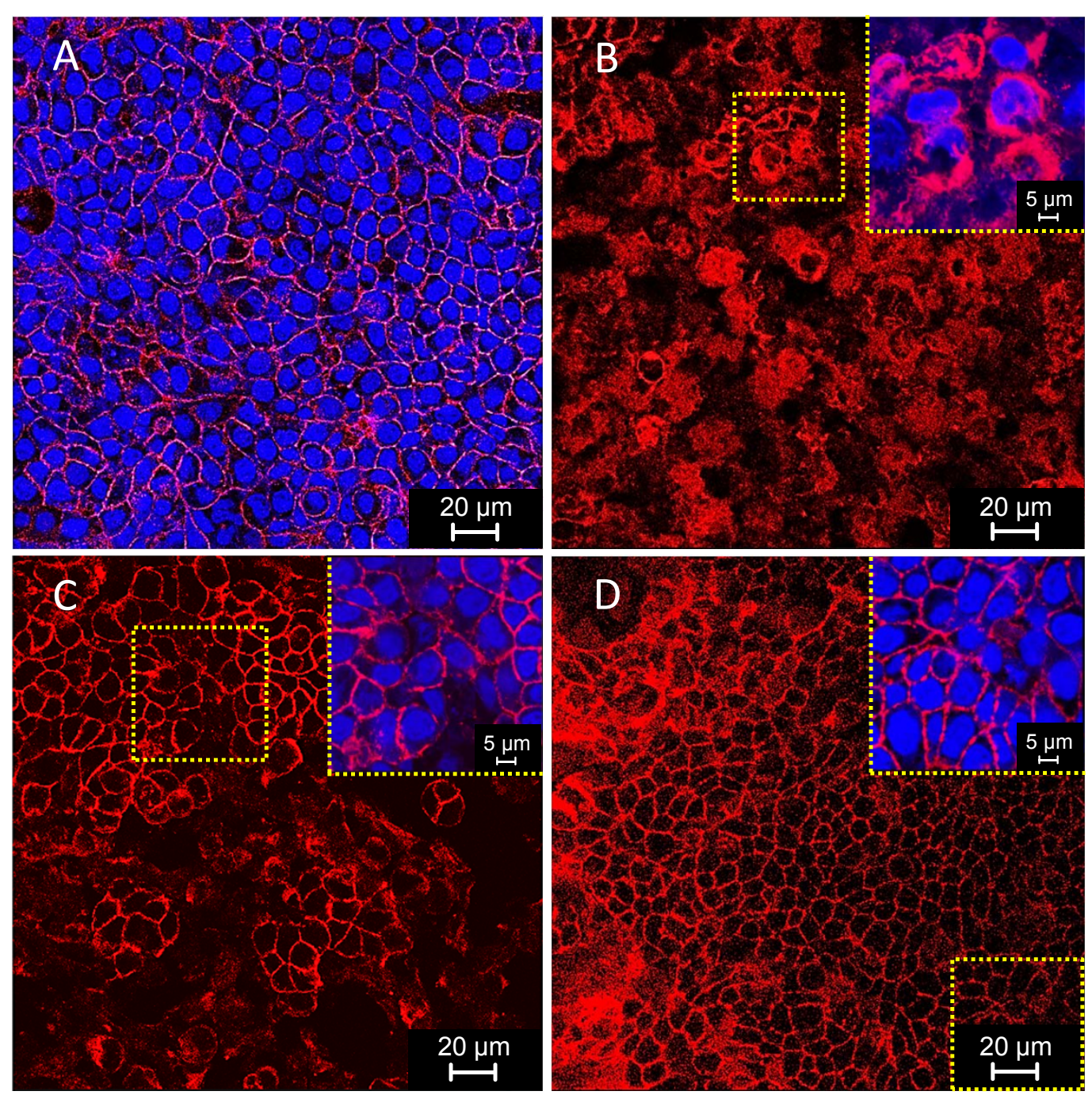

Figure 4 HT29-MTX monolayer integrity in complex colonic environments is affected by Salmonella infection and probiotic treatments. Tight junctions (in red) and nuclei (in blue) of HT29-MTX cells were stained with phalloidin and DAPI, respectively, after incubation for 90 min with distal reactor effluents of F1 retained at the end of (A, Stab) initial model stabilization, (B, Sal) Salmonella infection, (C, Ecol II) E. coli L1000 and (D, Bif I) B. thermophilum RBL67 periods. Tight junctions were highly disrupted after incubation with effluents from Salmonella infection (Sal) compared to initial model stabilization periods (Stab).

incubation with effluents of all reactors from Salmonella infection periods (Sal) were significantly lower $(P<$ 0.0001 , Table 1 ), with a mean decrease of $40 \pm 4 \%$ (Figure 2D). This effect on cell integrity was confirmed by confocal microscopy analysis which demonstrated highly disrupted tight junctions after Salmonella infection for distal reactor (R3) effluents of F1 (Figure 4B) compared to initial model stabilization periods (Figure 4A).

\section{E. coli L1000 stimulates Salmonella growth yet reduces invasion in the distal colon region}

E. coli L1000 established itself in the three-stage model at low levels with slightly but non-significantly higher numbers measured in R3 $\left(4.9 \pm 0.9 \log _{10} \mathrm{MCN} / \mathrm{ml}\right)$ compared to $\mathrm{R} 1\left(4.5 \pm 0.6 \log _{10} \mathrm{MCN} / \mathrm{ml}\right)$ and $\mathrm{R} 2(4.3$ $\pm 0.6 \log _{10} \mathrm{MCN} / \mathrm{ml}$; Figure 2A).

As shown previously [15], the addition of E. coli L1000 beads to the intestinal fermentation model enhanced Salmonella growth in all colon reactors compared to initial Salmonella infection periods (Sal; Figure 2A). However, significantly lower Salmonella invasion ratios were measured with transverse and distal reactor effluents (Figure 2B) in comparison with initial Salmonella stabilization periods (Sal). Concomitantly, Salmonella adhesion ratios remained stable in R3 (Figure 2B), however the efficiency of cell-associated Salmonella to invade HT29-MTX cells (Figure 2C) decreased significantly. The second addition of E. coli L1000 (Ecol II) 
had no further effects on Salmonella adhesion and invasion ratios in $\mathrm{R} 1$ and $\mathrm{R} 3$. However, a significantly enhanced $(P=0.0004)$ Salmonella invasion ratio was measured with transverse reactor effluents (Figure 2B) compared to the first E. coli L1000 period (Ecol I), which was accompanied by a significant increase in invasion efficiency (Figure 2C).

Similar mean TER values were measured with effluents from first E. coli L1000 (Ecol I) and Salmonella colonization (Sal) periods for all reactors (Table 1, Figure 2D), despite significantly higher Salmonella counts $(P<0.01)$ after the addition of $E$. coli L1000 (Figure $2 \mathrm{~A})$. TER significantly $(P>0.05)$ decreased by $19 \%$ and $26 \%$ with transverse and distal reactor effluents respectively (Figure 2D) after the second addition of $E$. coli L1000 (Ecol II) compared to the previous period (Ecol I) while Salmonella counts did not change for the two $E$. coli periods (Figure 2A).

\section{B. thermophilum RBL67 exerts a protective effect on epithelial integrity in highly infected environments}

B. thermophilum RBL67 colonized all reactors of the two three-stage fermentation models, reaching high counts of $6.8 \pm 0.5,6.4 \pm 0.4$ and $6.5 \pm 0.3 \log _{10} \mathrm{MCN} /$ $\mathrm{ml}$ in R1, R2 and R3, respectively (Bif; Figure 2A). Addition of B. thermophilum RBL67 beads increased Salmonella counts in R1 compared to the previous E. coli L1000 treatment (Ecol II, Figure 2C). However, Salmonella invasion efficiency did not change for any of the reactors and the invasion ratio measured with transverse reactor samples significantly decreased during Bif compared to Ecol II periods (Figure 2B).

B. thermophilum RBL67 addition (Bif) significantly ( $P$ $=0.0001)$ increased the mean TER measured across HT29-MTX cell monolayers applied with effluents of all reactors by $58 \pm 17 \%$ compared to previous $E$. coli L1000 period (Ecol II, Figure 2D). Mean TER measured after $24 \mathrm{~h}$ of incubation with effluents from proximal reactors $\left(130 \pm 47 \Omega \mathrm{cm}^{2}\right)$ was similar $(P>0.05)$ to initial model stabilization periods (Stab) before Salmonella infection $\left(127 \pm 23 \Omega \mathrm{cm}^{2}\right.$; Table 1$)$. Confocal microscopy analysis revealed high integrity of intracellular junctions upon application of distal colon reactor effluents of F1 after addition of B. thermophilum RBL67 (Figure 4D) despite high Salmonella counts (6.4 \pm 0.6 $\left.\log _{10} \mathrm{cfu} / \mathrm{ml}\right)$.

\section{Inulin stimulates $B$. thermophilum RBL67 growth but increases Salmonella invasion in proximal colon environments}

Addition of inulin induced a significant $(P=0.022)$ increase in Salmonella counts (Figure 2A) in R3 compared to previous B. thermophilum RBL67 periods (Bif). Furthermore a pronounced enhancement of $B$. thermophilum RBL67 growth (Figure 2A) and an increase in SCFA concentrations and butyrate ratios (Table 1) occurred in all reactors. Inulin supplementation in R1 was accompanied by a significant $(P=0.024)$ increase in the efficiency of Salmonella to invade HT29MTX cells compared to the previous $B$. thermophilum RBL67 period (Bif). This effect was not significant for transverse and distal reactor samples. Inulin treatment also induced a $25 \%$-decrease $(P=0.088)$ in TER after 1 $3 \mathrm{~h}$ of incubation for effluents of R1 compared to the previous $B$. thermophilum RBL67 periods (Table 1), while a similar but less pronounced tendency was observed for transverse and distal reactors.

\section{Discussion}

Accurate assessment of probiotic-mediated anti-Salmonella activities is complicated by the fact that mechanisms involved in enteric protection are the function of many probiotic features. Various interactions take place in complex gut environments, including competition for substrates, direct antagonism by the production of inhibitory substances (e.g. SCFA or bacteriocins), competitive exclusion, and potentially host-mediated effects such as improved barrier function and altered immune response $[5,28,29]$. It is therefore crucial to consider microbe-microbe as well as host-microbe interactions for the development of probiotics with targeted efficacy. Beyond animal in vivo models, combinatorial in vitro systems using both gut fermentation and cell models are an integral component in system biology approaches aimed at developing new probiotics [6]. For example, the dynamic TNO-gastrointestinal system (TIM-1) of the human small intestine combined with the Caco-2 cell model was used to investigate the digestive stability and intestinal absorption of lycopene and $\alpha$-tocopherol [7] Furthermore, adhesion to and cytokine expression of Caco-2 cells was assessed using bacterial cultures, including the probiotic strain Bifidobacterium longum DD2004, obtained from a three-stage continuous-culture system (CCS) simulating the proximal and distal large intestine [8]. Results clearly indicate that application of fermentation effluents to intestinal cells represents a valuable platform for assessing epithelial responses as a function of in vitro fermentative processes and microbial interactions. In this study, a three-stage continuous intestinal fermentation model closely mimicking conditions in the proximal, transverse and distal colon regions and inoculated with immobilized child feces was used to generate a complex microbiota. For the first time, we report the effects of Salmonella in a complex gut microbiota containing metabolites and grown under environmental conditions of the different sections of the colon, on mucus-secreting intestinal HT29-MTX cells. This combined model approach was used to assess host- 
protecting, anti-Salmonella activities of probiotic and prebiotic combinations.

Mean invasion efficiencies of $S$. Typhimurium N-15 into HT29-MTX cells measured in colonic effluents were up to 50 -fold lower compared to values measured in simple experimental conditions of a single Salmonella strain in DMEM, reflecting different microbe cell interactions in simple systems compared to environments with a complex gut microbiota [24]. Bacterial interactions occurring at the brush-border of HT29-MTX cells may enhance barrier function and diminish Salmonella invasion capacity, through the presence of a complex host microbiota, specific metabolites, as well as competition for adhesion sites. SCFAs at physiological concentrations are known to induce a concentrationdependent, reversible change in cellular permeability in vitro [25,30]. A higher concentration of total SCFAs in fecal water of adults applied to Caco- 2 cells was shown to be associated with an increase in TER in comparison to fecal water obtained from elderly subjects containing lower SCFA concentrations which negatively affected epithelial barrier function [31]. Our results obtained with effluents sampled at the end of model stabilization periods (Stab) were in accordance with these findings. Indeed, a generally higher TER across HT29-MTX cell monolayers was measured after $24 \mathrm{~h}$ of incubation for transverse and distal reactor samples with a high concentration of SCFAs accumulating in the in vitro model due to the lack of absorption, compared to samples from the proximal reactor. In general, lower TER values were measured during all experimental periods and for all reactors upon effluent exposure for $24 \mathrm{~h}$ compared to 1-3 h. As reactor effluents contain a dense and active microbiota, bacterial fermentation and $\mathrm{pH}$ reduction can occur during intestinal cell incubation which can negatively affect cell viability thus epithelial integrity [23].

Salmonella invasion is influenced by environmental factors such as $\mathrm{pH}$ or SCFA concentrations. Upon infection Salmonella invasion was generally higher in distal reactors ( $\mathrm{pH}$ 6.7) compared to proximal ( $\mathrm{pH}$ 5.7) and transverse $(\mathrm{pH} 6.2)$ reactors and inversely related to SCFA concentrations. These results are consistent with findings of Durant et al. [32], demonstrating that Salmonella entry into HEp-2 cells was higher at pH 7 compared to pH 6 in the presence of $80 \mathrm{mM}$ acetate, 40 $\mathrm{mM}$ propionate and $20 \mathrm{mM}$ butyrate. A lower percentage of cell-association and invasion was observed as the concentration of each SCFA increased at pH 6 but not at pH 7 [32]. Salmonella invasion into intestinal cells is known to be associated with a rapid disruption of epithelial integrity caused by structural modifications of intercellular junctions that can be assessed by TER measurements $[8,33,34]$. In this study, we effectively demonstrated that effluents obtained from three-stage in vitro colonic fermentation models of Salmonella infection and applied directly on confluent and fully differentiated HT29-MTX cells induces a large and significant decrease of TER after $1 \mathrm{~h}$ of incubation, compared to non-infected effluents (Figure 3). Visualization of tight junctions by phalloidin staining revealed that intracellular junctions of HT29-MTX cells were not affected by the gut microbiota produced during initial model stabilization (Stab, Figure 4A) but were highly disrupted in the presence of Salmonella (Sal, Figure 4B). This is in accordance with results published by Jepson et al. [35] where incubation of MDCK monolayers with S. typhimurium SL1344 for 60 min was accompanied by a disruption of intracellular junctions.

Addition of E. coli L1000 enhanced Salmonella growth in all reactors although the efficiency of Salmonella in invading HT29-MTX cells significantly decreased in distal reactor (R3) samples. After the addition of B. thermophilum RBL67, the invasion efficiency of Salmonella decreased most in proximal reactors (R1), despite higher Salmonella counts compared to previous Ecol II periods. These results may reflect the influence of environmental requirements for optimal growth of the tested probiotics. B. thermophilum RBL67 is acid tolerant and a competitive bacteriocinogenic bacteria $[15,18]$, a trait likely advantageous for competing with other members of the bacterial ecosystem present in proximal colon reactors at $\mathrm{pH}$ 5.7. Indeed, B. thermophilum RBL67 best colonized and reduced Salmonella invasion into HT29-MTX cells at $\mathrm{pH} 5.7$ with proximal reactor samples, while $E$. coli L1000 was more competitive at pH 6.6 in distal colon reactors.

The presence of E. coli L1000 in the fermentation model not only enhanced Salmonella growth but also induced further disruption of epithelial integrity, a finding which was unexpected. A similar decrease in TER was observed for T84 cells when preventively incubated with E. coli Nissle 1917 before addition of S. dublin [36]. In contrast, TER values and epithelial integrity after B. thermophilum RBL67 addition were significantly enhanced in all reactors of both models although Salmonella counts were very high. Several studies reported that live Gram-positive probiotics are able to enhance monolayer barrier function and protect cultured epithelial cells from the effects of infection with invasive pathogens. Preventive treatments with Lactobacillus acidophilus and Streptococcus thermophilus, for example, were shown to prevent the enteroinvasive Escherichia coli (EIEC)-induced decrease in TER of HT29/cl 19A cell monolayers [37]. Bifidobacterium infantis and Bifidobacterium breve of the probiotic cocktail VSL\#3, were shown to improve epithelial integrity of T84 cells and resistance to Salmonella invasion [38]. It was suggested that Gram-positive and Gram-negative probiotics use 
different mechanisms to beneficially modulate the intestinal epithelium and to mediate protection against Salmonella [36]. Indeed, the ability of E. coli Nissle 1917 and the probiotic mixture VSL\#3 to diminish Salmonella dublin-induced death of T84 cells was related to the induction of IL-8 secretion by the Gram-negative probiotic, while the Gram-positive probiotic mixture was shown to prevent pathogen-induced decrease in TER and stabilize tight junctions.

Among SCFAs, a special function is assigned to butyrate. In the gut lumen, butyrate is used by epithelial cells as an energy source whereas in tumor cells (e.g. HT29-MTX) butyrate reduces survival by inducing apoptosis and inhibiting proliferation $[19,39,40]$ with concentrations $\geq 8 \mathrm{mM}$ being shown to reduce TER of Caco-2 cells [41]. A similar effect was observed in this study. Inulin induced a strong bifidogenic effect and a shift in SCFA ratios, with a strong increase in butyrate concentrations (Table 1), accompanied by a decrease in TER.

\section{Conclusions}

Our results highlight the benefits of combining suitable cellular and colonic fermentation models to evaluate host protection activity of probiotics during Salmonella infection in the presence of commensal gut organisms, providing efficient tools for mechanistic studies in vitro which may enhance preclinical development of new antimicrobials. The application of a complex microbiota produced in an in vitro fermentation model to HT29MTX cells revealed that optimal environmental conditions and the impact on Salmonella infectivity and intestinal epithelial integrity differed for both probiotic strains tested. E. coli L1000 remained at low levels but preferentially colonized the simulated distal colon and also stimulated Salmonella growth which was accompanied by a significant disruption of epithelial integrity. In contrast, $B$. thermophilum RBL67 was very competitive and established itself at high levels preferentially in proximal colon reactors. Its presence induced a high increase in TER after $24 \mathrm{~h}$ of incubation in all reactors and both models to levels similar to that measured before Salmonella addition. Additional studies examining cellular immune responses, including utilizing fecal material from other donors to account for differences in individual gut ecosystems, are necessary in further elucidating the mechanisms of B. thermophilum RBL67 and E. coli L1000 for treatment of Salmonella infections prior to large-scale and costly in vivo trials.

\section{Methods}

Bacterial strains

Salmonella enterica spp. enterica serovar Typhimurium N-15 (S. Typhimurium N-15) was isolated in 2007 from an infected person in Switzerland and obtained from the National Center for Enteropathogenic Bacteria (NENT, Luzern, Switzerland). It was routinely cultivated in tryptic soy broth (TSB, Difco, Basel Switzerland) at $37^{\circ} \mathrm{C}$ for $18 \mathrm{~h}$.

E. coli L1000 wt, producing microcin B17 [16], was kindly provided by Hans-Dieter Grimmecke (Laves-Arzneimittel GmbH, Schötz, Switzerland). A mutant strain lacking microcin B17-phenotype (E. coli L1000 MccB17) was also used [15]. B. thermophilum RBL67, initially isolated from baby feces [42], was obtained from our culture collection.

\section{Intestinal in vitro colonic fermentations}

Intestinal colonic fermentations were performed as previously reported [15]. In brief, two three-stage continuous in vitro fermentation models (F1 and F2) inoculated with the same immobilized child fecal microbiota were infected with $S$. Typhimurium N-15. These models were operated in parallel for 65 days to test and compare the effects of treatments with probiotic E. coli L1000 wt and MccB17-, followed by B. thermophilum RBL67, and prebiotic inulin, on gut microbiota composition, activity, probiotic growth and Salmonella colonization [15]. Specific retention times ( $\mathrm{RT}$ ) and $\mathrm{pH}$ were applied to the three reactors of each model corresponding to the physiological conditions in child proximal (R1), transverse (R2) and distal (R3) colons: $\mathrm{RT}=5 \mathrm{~h}$ and $\mathrm{pH} 5.7$ for $\mathrm{R} 1, \mathrm{RT}=10 \mathrm{~h}$ and $\mathrm{pH} 6.2$ for $\mathrm{R} 2$, and $\mathrm{RT}=10 \mathrm{~h}$ and $\mathrm{pH} 6.6$ for R3, respectively [43,44].

Continuous fermentations were divided into six consecutive experimental periods illustrated in Figure 1 and presented in detail by Zihler et al. [15]. Briefly, the first model F1 used to test E. coli L1000 wt, included the following conditions: (1) system stabilization [Stab, 10 days], (2) S. Typhimurium N-15 beads addition to R1 to induce Salmonella infection [Sal, 9 days], (3) first E. coli L1000 $w t$ beads addition to R1 [Ecol I, 14 days], (4) second E. coli L1000 wt beads addition to R3 [Ecol II, 8 days], (5) first $B$. thermophilum RBL67 beads addition to R1 [Bif, 11 days], and (6) second B. thermophilum RBL67 beads addition to R1 [Bif II, 10 days]. In the second model F2 E. coli L1000 wt was replaced by E. coli L1000 MccB17- to assess the effect of microcin B17 phenotype. Similar periods as F1 were tested except for the last period (6) during which prebiotic inulin was tested [Inulin, 10 days].

Effluents $(13 \mathrm{ml})$ were collected daily from each reactor of the two models and processed within $1 \mathrm{~h}$ for the enumeration of S. Typhimurium N-15 (selective plating), quantification of main bacterial populations (real-time qPCR analyses), and metabolic analysis [15]. Fresh effluents were also directly applied on intestinal HT29-MTX cells. 


\section{Bacterial enumeration}

\section{Salmonella enumeration by plate counts}

Salmonella viable cell counts were measured during the last 3 days of each experimental period corresponding to pseudo-steady-state conditions. Effluent samples were serially diluted 10 -fold in peptone water $(0.1 \%, \mathrm{pH} 7.0)$ and plated in duplicate on CHROMAgar ${ }^{\mathrm{TM}}$ Salmonella (Becton Dickinson AG, Allschwil, Switzerland). Plates were incubated at $37^{\circ} \mathrm{C}$ for $48 \mathrm{~h}$.

\section{E. coli $L 1000$ and B. thermophilum RBL67 enumeration by real-time $q P C R$ analysis}

E. coli L1000 and B. thermophilum RBL67 concentrations in reactor effluents were estimated by real-time qPCR analysis as described before [15]. Mean copy numbers $(\mathrm{MCN} / \mathrm{ml})$ were calculated for the last 3 days of each experimental period of F1 and F2.

\section{Metabolite analysis}

Short-chain fatty acids [SCFA: acetate (A), propionate (P) and butyrate (B)] concentrations in effluent samples were determined in duplicate by high-performance liquid chromatography (HPLC) analysis [12].

\section{Cell cultures}

The human mucus-secreting intestinal colon cancer cell line HT29-MTX [45], obtained after long-term treatment of human carcinoma HT-29 cells with the anti-cancer drug methotrexate [46], was kindly provided by Dr. Thécla Lesuffleur (INSERM, Lille, France). Cells were routinely maintained at $37^{\circ} \mathrm{C}$ in a humidified incubator $\left(10 \% \mathrm{CO}_{2}\right)$ in complete Dulbecco's Modified Eagle medium Glutamax (DMEM; Invitrogen AG, Basel, Switzerland) supplemented with $10 \%(\mathrm{~V} / \mathrm{V})$ fetal bovine serum (FBS; Invitrogen AG) and $1 \%(\mathrm{~V} / \mathrm{V})$ antibiotics (10'000 $\mathrm{U} / \mathrm{ml}$ penicillin $+10^{\prime} 000$ $\mu \mathrm{g} / \mathrm{ml}$ streptomycin; Invitrogen AG). For invasion assays, cells were seeded in 24-well tissue culture plates $\left(2 \mathrm{~cm}^{2}\right.$ well $^{-1}$; Bioswisstec AG, Schaffhausen, Switzerland) at a concentration of $4 \times 10^{4}$ cells per well and cultivated for 21 days to reach complete confluence and differentiation. The medium was replaced every 2 days and cell viability was determined by tryptan blue staining $(0.1 \%(\mathrm{~V} / \mathrm{V})$ in 10 $\mathrm{mM}$ phosphate buffered saline (PBS), $\mathrm{pH}$ 7.3). DMEM without antibiotics was used for the last medium change before using the cells for invasion assays.

For transepithelial electrical resistance (TER) measurements, HT29-MTX cells were seeded in cell culture inserts with a $0.45 \mu \mathrm{m}$ filter membrane and a $0.7 \mathrm{~cm}^{2}$ surface area (24-well culture plate, Millipore AG, Zug, Switzerland) at a concentration of $2.3 \times 10^{5}$ cells per insert and cultivated as described above.

\section{Invasion assays}

A gentamicin-based assay, as described by Steele-Mortimer et al. (2008) but with some modifications, was performed to determine the capacity of Salmonella present in reactor effluents to invade HT29-MTX cells. Briefly, $1 \mathrm{ml}$ effluents obtained during the last 3 days of each fermentation period from proximal (R1), transverse (R2) and distal (R3) colon reactors were applied directly in duplicate on cell layers of three consecutive passages and incubated at $37^{\circ} \mathrm{C}$ for $90 \mathrm{~min}$. To kill non-invading bacteria, cell layers were washed twice with $250 \mu \mathrm{l}$ PBS before adding $250 \mu \mathrm{l}$ DMEM supplemented with 150 $\mu \mathrm{g} / \mathrm{ml}$ gentamicin (Sigma-Aldrich Chemie $\mathrm{GmbH}$, Buchs, Switzerland) per well followed by an additional incubation period for $60 \mathrm{~min}$ at $37^{\circ} \mathrm{C}$. After a further washing step with PBS, $250 \mu \mathrm{l}$ Trypsin-EDTA (1X, Invitrogen) were added followed by another incubation for $10 \mathrm{~min}$. Finally, cells were disrupted by adding $250 \mu \mathrm{l}$ $0.1 \%(\mathrm{~V} / \mathrm{V})$ Triton X-100 (Sigma) per well and incubating for $10 \mathrm{~min}$ before samples were collected for enumeration of invaded Salmonella. The same protocol but without gentamicin treatments was used for the determination of cell-associated Salmonella (accounting for both invasive and adherent bacteria). The number of adhered Salmonella was then calculated from the difference of cell-associated to invaded bacteria. Adhesion and invasion ratios were expressed as the percentage of adhered and invaded bacteria, respectively, related to the total number of Salmonella present in effluents. Invasion efficiency measured during different probiotic and prebiotic treatments was expressed as the percentage of invaded bacteria related to the number of cell-associated Salmonella.

The same protocol was used to measure the invasion efficiency of $S$. Typhimurium N-15 in pure culture when applied in artificial DMEM medium. Therefore, the pellet of an overnight culture of Salmonella obtained by centrifugation (8000 g, $5 \mathrm{~min}$ ) was diluted in DMEM to reach a concentration of $1.0 \times 10^{7} \mathrm{cfu} / \mathrm{ml} .125 \mu \mathrm{l}$ of this bacterial suspension was added in duplicate to cell monolayers that corresponded to a Salmonella concentration $\left(1.3 \times 10^{6} \mathrm{cfu} / \mathrm{ml}\right)$ measured in effluents from the two models during Sal periods.

\section{Transepithelial electrical resistance (TER) measurements}

TER measurements were performed to estimate the degree of cell monolayer's integrity loss that occurs during Salmonella infection due to disruption of tight junctions [33]. To measure the epithelial integrity of HT29MTX cells, $400 \mu \mathrm{l}$ of effluent was applied directly to the apical compartment of PBS-washed HT29-MTX cell culture inserts that were prepared as previously described. TER measurements were performed before effluent application and after 1, 2, 3 and $24 \mathrm{~h}$ of incubation at $37^{\circ} \mathrm{C}$. The resistance of cell layers was calculated by subtracting the intrinsic resistance of the filter insert alone from the total measured resistance (filter insert 
plus cell layer and effluents) and expressed as $\Omega$ per $\mathrm{cm}^{2}$ surface area. The same protocol was used to measure the influence of $S$. Typhimurium N-15 on TER of HT29-MTX cells in artificial DMEM medium as presented before.

\section{Microscopic analysis of tight junctions}

To visualize the effects of Salmonella infection on cell monolayer integrity before and during probiotic treatments, tight junctions and the nucleus of confluent HT29-MTX cells were fluorescently stained according to previous studies $[35,47]$.

Briefly, HT29-MTX cells were seeded at $9.6 \times 10^{4}$ cells $/ \mathrm{ml}$ on a coverslip in a 6 -well tissue culture plate and cultured to confluence before incubation with $1 \mathrm{ml}$ of distal colon reactor (R3) effluents from the last day of different treatment periods of F1. DMEM-high glucose without Phenol red (Invitrogen AG, Basel, Switzerland) supplemented with $10 \%(\mathrm{~V} / \mathrm{V})$ fetal bovine serum (FBS; Invitrogen $A G$ ) and without antibiotics was used for the last medium change before invasion assays. After incubation of $1 \mathrm{ml}$ effluent for $90 \mathrm{~min}$, cells were washed thrice with PBS and fixed overnight in $1 \mathrm{ml}$ per well of a chilled 4\% (V/V) formaldehyde (Sigma-Aldrich Chemie $\mathrm{GmbH}$, Buchs, Switzerland) in PBS solution. After a second washing step (3 times with PBS), cells were permeabilized by treating them with $200 \mu \mathrm{l}$ of $0.1 \%$ Triton X100 in PBS for $3 \mathrm{~min}$ at room temperature. After a third washing step (3 times with PBS), cells were treated with $1 \mathrm{ml}$ of $3 \%(\mathrm{~V} / \mathrm{V})$ albumin bovine serum (BSA, SigmaAldrich Chemie $\mathrm{GmbH}$ ) in PBS to prevent non-specific binding of fluorescent dyes. Tight junctions were stained for $40 \mathrm{~min}$ with $1 \mathrm{ml}$ of a 1:200 PBS-diluted stock solution $(0.1 \mathrm{mg} / \mathrm{ml})$ of phalloidin-tetramethylrhodamine $B$ isothiocyanate (phalloidin-TRITC, Sigma-Aldrich Chemie $\mathrm{GmbH}$ ) in methanol, while nuclei were stained for 3 min with $1 \mathrm{ml}$ of a 1:100 PBS-diluted stock solution (5 $\mathrm{mg} / \mathrm{ml}$ ) of 4', 6-diamidino-2-phenylindole (DAPI, Sigma-Aldrich Chemie $\mathrm{GmbH}$ ) in ultrapure water. After a last washing step, coverslips were mounted inverted on a coverglas by applying one drop of the embedding media Glycergel (DakoCytomation; Glostrup, Denmark). Microscopic analyses were performed with a confocal laser scanning microscope (SP 2, Leica Microsystems, Mannheim, Germany). Different series of images were obtained and stacked by using the Imaris 7 software (Bitplane AG, Zürich, Switzerland).

\section{Statistical analysis}

All statistical analyses were performed using JMP 8.0 for Windows (SAS Institute Inc., Cary, NC, USA). Bacterial counts as well as adhesion and invasion data were $\log 10$-transformed to stabilize the variance and normalize residuals values for variance homogeneity.
A one-way analysis of variance (ANOVA) was performed to compare the effects of two consecutive treatments on mean Salmonella counts, adhesion and invasion capacities, as well as percentage changes in invasion and adhesion ratios, invasion efficiencies and transepithelial electrical resistance (TER). Measurements during the last 3 days of each fermentation period corresponding to a pseudo-steady-state were used as repetition. Salmonella counts, invasion and adhesion ratios, as well as invasion efficiency and TER measured during the last 3 days of each experimental period were not significantly different for F1 and F2, which were inoculated with the same child fecal microbiota immobilized in beads. Therefore, data obtained during system stabilization (Stab), Salmonella colonization (Sal) as well as E. coli L1000 (Ecol) and B. thermophilum RBL67 (Bif) treatment periods of F1 and F2 were used as independent replicates. TER data measured after 1,2 and $3 \mathrm{~h}$ of incubation were not significantly different $(P>0.05)$. Therefore, mean TER values for the three incubation times were reported. Treatment means were compared using the Tukey-Kramer-HSD test with probability levels of $P<0.05$ and $P<0.01$.

\section{Abbreviations}

A: Acetate; B: Butyrate; cfu: colony forming units; DMEM: Dulbecco's Modified Eagle medium Glutamax; F1: Continuous three-stage fermentation system 1; F2: Continuous three-stage fermentation system 2; HPLC: HighPerformance Liquid Chromatography; MCN: Mean copy numbers; MRS: De Man: Rogosa and Sharpe; P: Propionate; PBS: Phosphate buffered saline; qPCR: Quantitative Polymerase Chain Reaction; R1: Fermentation reactor simulating proximal colon conditions; R2: Fermentation reactor simulating transverse colon conditions; R3: Fermentation reactor simulating distal colon conditions; RT: Retention time; SCFAs: Short-chain fatty acids; TER: Transepithelial electrical resistance; TSB: Tryptic soy broth.

\section{Acknowledgements}

We thank the Center for Microscopy and Image Analysis (University of Zurich, Zurich, Switzerland) for assistance with microscopic analyses. This study was supported by a grant from the Swiss National Science Foundation (SNF, project number: 3100170-114028).

\section{Authors' contributions}

$A Z, M G, C C$ and $C L$ conceived the study. $A Z$ and $M G$ carried out the experiments. $A Z, M G, C L$ and $C C$ analyzed results and drafted the manuscript. All authors read and approved the final manuscript.

Received: 18 August 2011 Accepted: 15 December 2011 Published: 15 December 2011

\section{References}

1. Gaskins HR, Croix JA, Nakamura N, Nava GM: Impact of the intestinal microbiota on the development of mucosal defense. Clin Infect Dis 2008, 46(Suppl 2):S80-S86, discussion S144-S151.

2. Bernet MF, Brassart D, Neeser JR, Servin AL: Lactobacillus acidophilus LA 1 binds to cultured human intestinal cell lines and inhibits cell attachment and cell invasion by enterovirulent bacteria. Gut 1994, 35:483-489.

3. Viswanathan VK, Hodges $K$, Hecht G: Enteric infection meets intestinal function: how bacterial pathogens cause diarrhoea. Nat Rev Microbiol 2009, 7:110-119.

4. Claesson MJ, O'Sullivan O, Wang Q, Nikkila J, Marchesi JR, Smidt H, de Vos WM, Ross RP, O'Toole PW: Comparative analysis of pyrosequencing 
and a phylogenetic microarray for exploring microbial community structures in the human distal intestine. PLoS One 2009, 4:e6669.

5. Collado MC, Isolauri E, Salminen S, Sanz Y: The impact of probiotic on gut health. Curr Drug Metab 2009, 10:68-78.

6. Payne NA, Zihler A, Chassard C, Lacroix C: Advances and perspectives in in vitro human gut fermentation modeling. Trends Biotechnol 2011, doi:10.1016/j.tibtech.2011.06.2011.

7. Deat E, Blanquet-Diot S, Jarrige JF, Denis S, Beyssac E, Alric M: Combining the dynamic TNO-gastrointestinal tract system with a Caco-2 cell culture model: application to the assessment of lycopene and alpha-tocopherol bioavailability from a whole food. J Agric Food Chem 2009, 57:11314-11320

8. Bahrami B, Child MW, Macfarlane S, Macfarlane GT: Adherence and cytokine induction in Caco-2 cells by bacterial populations from a threestage continuous-culture model of the large intestine. Appl Environ Microbiol 2011, 77:2934-2942.

9. Cinquin C, Le Blay G, Fliss I, Lacroix C: Immobilization of infant fecal microbiota and utilization in an in vitro colonic fermentation model. Microb Ecol 2004, 48:128-138.

10. Cinquin C, Le Blay G, Fliss I, Lacroix C: New three-stage in vitro model for infant colonic fermentation with immobilized fecal microbiota. FEMS Microbiol Ecol 2006, 57:324-336.

11. Cinquin C, Le Blay G, Fliss I, Lacroix C: Comparative effects of exopolysaccharides from lactic acid bacteria and fructo-oligosaccharides on infant gut microbiota tested in an in vitro colonic model with immobilized cells. FEMS Microbiol Ecol 2006, 57:226-238.

12. Cleusix V, Lacroix C, Vollenweider S, Le Blay G: Glycerol induces reuterin production and decreases Escherichia coli population in an in vitro model of colonic fermentation with immobilized human feces. FEMS Microbiol Ecol 2008, 63:56-64.

13. Le Blay G, Rytka J, Zihler A, Lacroix C: New in vitro colonic fermentation model for Salmonella infection in the child gut. FEMS Microbiol Ecol 2009, 67:198-207.

14. Le Blay G, Chassard C, Baltzer S, Lacroix C: Set up of a new in vitro model to study dietary fructans fermentation in formula-fed babies. Br J Nutr 2010, 103:403-411

15. Zihler A, Gagnon M, Chassard C, Hegland A, Stevens MJ, Braegger CP, Lacroix C: Unexpected consequences of administering bacteriocinogenic probiotic strains for Salmonella populations, revealed by an in vitro colonic model of the child gut. Microbiology 2010, 156:3342-3353.

16. Zihler A, Le Blay G, de Wouters T, Lacroix C, Braegger CP, Lehner A, Tischler $P$, Rattei $T$, Hachler H, Stephan R: In vitro inhibition activity of different bacteriocin-producing Escherichia coli against Salmonella strains isolated from clinical cases. Lett Appl Microbiol 2009, , 49: 31-38.

17. von Ah U: Identification of Bifidobacterium thermophilum RBL67 isolated from baby faeces and partial purification of its bacteriocin. PhD thesis Diss Nr 16927, Swiss Federal Institute of Technology Zurich (ETHZ), Zurich, Switzerland; 2006.

18. von Ah U, Mozzetti V, Lacroix C, Kheadr EE, Fliss I, Meile L: Classification of a moderately oxygen-tolerant isolate from baby faeces as Bifidobacterium thermophilum. BMC Microbiol 2007, 7:79.

19. Mennigen $\mathrm{R}$, Bruewer M: Effect of probiotics on intestinal barrier function. Ann N Y Acad Sci 2009, 1165:183-189.

20. Gagnon M, Zihler A, Chassard C, Lacroix C: Ecology of probiotics and enteric protection. In Probiotic Bacteria and Enteric Infections-Cytoprotection by probiotic bacteria. Volume 1. Edited by: Malago JJ, Koninkx JFJG, Marinsek-Logar R. Springer Science + Business Media B.V.; 2011:65-85.

21. Weinstein DL, O'Neill BL, Hone DM, Metcalf ES: Differential early interactions between Salmonella enterica serovar Typhi and two other pathogenic Salmonella serovars with intestinal epithelial cells. Infect Immun 1998, 66:2310-2318

22. Rabot S, Rafter J, Rijkers GT, Watzl B, Antoine JM: Guidance for substantiating the evidence for beneficial effects of probiotics: impact of probiotics on digestive system metabolism. J Nutr 2010, 140:677S-689S.

23. Alemka A, Clyne M, Shanahan F, Tompkins T, Corcionivoschi N, Bourke B: Probiotic colonization of the adherent mucus layer of HT29MTXE12 cells attenuates Campylobacter jejuni virulence properties. Infect Immun 2010, , 78: 2812-2822.

24. Cencic A, Langerholc T: Functional cell models of the gut and their applications in food microbiology-a review. Int J Food Microbiol 2010, 141(Suppl 1):S4-S14.
25. Bahrami B, Macfarlane $S$, Macfarlane GT: Induction of cytokine formation by human intestinal bacteria in gut epithelial cell lines. J Appl Microbiol 2011, 110:353-363.

26. Stoidis CN, Misiakos EP, Patapis P, Fotiadis Cl, Spyropoulos BG: Potential benefits of pro- and prebiotics on intestinal mucosal immunity and intestinal barrier in short bowel syndrome. Nutr Res Rev 2010, 1-9.

27. Rishi P, Pathak S, Ricke SC: Short chain fatty acids influence virulence properties of Salmonella enterica serovar Typhimurium. J Environ Sci Health B 2005, 40:645-657.

28. OToole PW, Cooney JC: Probiotic bacteria influence the composition and function of the intestinal microbiota. Interdiscip Perspect Infect Dis 2008, 2008:175285.

29. Corr SC, Hill C, Gahan CG: Chapter 1 Understanding the mechanisms by which probiotics inhibit gastrointestinal pathogens. Adv Food Nutr Res 2009, 56:1-15.

30. Kalliomaki M, Antoine JM, Herz U, Rijkers GT, Wells JM, Mercenier A: Guidance for substantiating the evidence for beneficial effects of probiotics: prevention and management of allergic diseases by probiotics. J Nutr 2010, 140:713S-721S.

31. Gill Cl, Heavey P, McConville E, Bradbury I, Fassler C, Mueller S, Cresci A, Dore J, Norin E, Rowland I: Effect of fecal water on an in vitro model of colonic mucosal barrier function. Nutr Cancer 2007, 57:59-65.

32. Durant JA, Lowry VK, Nisbet DJ, Stanker LH, Corrier DE, Ricke SC: Shortchain fatty acids affect cell-association and invasion of HEp-2 cells by Salmonella typhimurium. J Environ Sci Health B 1999, 34:1083-1099.

33. Sekelja M, Berget I, Naes T, Rudi K: Unveiling an abundant core microbiota in the human adult colon by a phylogroup-independent searching approach. ISME J 2011, 5:519-531.

34. Alemka A, Clyne M, Shanahan F, Tompkins T, Corcionivoschi N, Bourke B: Probiotic colonization of the adherent mucus layer of HT29MTXE12 cells attenuates Campylobacter jejuni virulence properties. Infect Immun 2010, 78:2812-2822.

35. Jepson MA, Collares-Buzato CB, Clark MA, Hirst BH, Simmons NL: Rapid disruption of epithelial barrier function by Salmonella typhimurium is associated with structural modification of intercellular junctions. Infect Immun 1995, 63:356-359.

36. Otte JM, Podolsky DK: Functional modulation of enterocytes by grampositive and gram-negative microorganisms. Am J Physiol Gastrointest Liver Physiol 2004, 286:G613-G626.

37. Resta-Lenert S, Barrett KE: Live probiotics protect intestinal epithelial cells from the effects of infection with enteroinvasive Escherichia coli (EIEC). Gut 2003, 52:988-997.

38. Madsen K, Cornish A, Soper P, McKaigney C, Jijon H, Yachimec C, Doyle J, Jewell $L$, De Simone C: Probiotic bacteria enhance murine and human intestinal epithelial barrier function. Gastroenterology 2001, 121:580-591.

39. de Los Reyes-Gavilan CG, Suarez A, Fernandez-Garcia M, Margolles A, Gueimonde M, Ruas-Madiedo P: Adhesion of bile-adapted Bifidobacterium strains to the HT29-MTX cell line is modified after sequential gastrointestinal challenge simulated in vitro using human gastric and duodenal juices. Res Microbiol 2011, 162:514-519.

40. Mirold S, Ehrbar K, Weissmuller A, Prager R, Tschape H, Russmann H, Hardt WD: Salmonella host cell invasion emerged by acquisition of a mosaic of separate genetic elements, including Salmonella pathogenicity island 1 (SPI1), SPI5, and sopE2. J Bacteriol 2001, 183:2348-2358.

41. Peng L, He Z, Chen W, Holzman IR, Lin J: Effects of butyrate on intestinal barrier function in a Caco-2 cell monolayer model of intestinal barrier. Pediatr Res 2007, 61:37-41.

42. Touré R, Kheadr E, Lacroix C, Moroni O, Fliss I: Production of antibacterial substances by bifidobacterial isolates from infant stool active against Listeria monocytogenes. J Appl Microbiol 2003, 95:1058-1069.

43. Fallingborg J, Christensen $L A$, Ingeman-Nielsen $M$, Jacobsen $B A$, Abildgaard K, Rasmussen HH, Rasmussen SN: Measurement of gastrointestinal $\mathrm{pH}$ and regional transit times in normal children. $J$ Pediatr Gastroenterol Nutr 1990, 11:211-214.

44. Wagener S, Shankar KR, Turnock RR, Lamont GL, Baillie CT: Colonic transit time-what is normal? J Pediatr Surg 2004, 39:166-169, discussion 166-169.

45. Lesuffleur T, Barbat A, Dussaulx E, Zweibaum A: Growth adaptation to methotrexate of HT-29 human colon carcinoma cells is associated with their ability to differentiate into columnar absorptive and mucussecreting cells. Cancer Res 1990, 50:6334-6343. 
46. Van de Wiele TR, Verstraete W, Siciliano SD: Polycyclic aromatic hydrocarbon release from a soil matrix in the in vitro gastrointestinal tract. J Environ Qual 2004, 33:1343-1353.

47. Kim KP, Loessner MJ: Enterobacter sakazakii invasion in human intestinal Caco- 2 cells requires the host cell cytoskeleton and is enhanced by disruption of tight junction. Infect Immun 2008, 76:562-570.

\section{doi:10.1186/1471-2180-11-264}

Cite this article as: Zihler et al:: Protective effect of probiotics on

Salmonella infectivity assessed with combined in vitro gut fermentationcellular models. BMC Microbiology 2011 11:264.

Submit your next manuscript to BioMed Central and take full advantage of:

- Convenient online submission

- Thorough peer review

- No space constraints or color figure charges

- Immediate publication on acceptance

- Inclusion in PubMed, CAS, Scopus and Google Scholar

- Research which is freely available for redistribution

Submit your manuscript at www.biomedcentral.com/submit
() Biomed Central 